\title{
Upregulation of Notch pathway molecules in oral squamous cell carcinoma
}

\author{
HIROSHI HIJIOKA ${ }^{1}$, TAKAO SETOGUCHI ${ }^{2}$, AKIHIKO MIYAWAKI ${ }^{1}$, HUI GAO $^{2}$, \\ TAKAYUKI ISHIDA $^{1}$, SETSURO KOMIYA ${ }^{2}$ and NORIFUMI NAKAMURA ${ }^{1}$ \\ ${ }^{1}$ Department of Oral and Maxillofacial Surgery, ${ }^{2}$ Department of Orthopaedic Surgery, Graduate School \\ of Medical and Dental Sciences, Kagoshima University, 8-35-1 Sakuragaoka, Kagoshima 890-8520, Japan
}

Received July 20, 2009; Accepted September 21, 2009

DOI: 10.3892/ijo_00000558

\begin{abstract}
The constitutive activation of the Notch pathway has been demonstrated in various types of malignancies. However, it remains unclear how the Notch pathway is involved in the pathogenesis of oral squamous cell carcinoma (OSCC). We investigated the expression of Notch pathway molecules in OSCC cell lines and biopsy specimens and examined the effect of Notch pathway inhibition. Reverse transcription-polymerase chain reaction revealed upregulation of Notch1, Notch2, Jagged1, HES1 and HEY1 in both OSCC cell lines and biopsy specimens. Immunohistochemical examination showed that the Notch intracellular domain accumulates in the nucleus of cells in OSCC cell lines and biopsy specimens. In addition, Jagged 1 is expressed in the cytoplasm of cells in OSCC cell lines and biopsy specimens. Furthermore, Notch pathway inhibition using a $\gamma$-secretase inhibitor prevented the growth of OSCC in vitro. These findings suggest that inhibition of the Notch pathway suppresses OSCC growth and may be a useful approach for the treatment of patients with OSCC.
\end{abstract}

\section{Introduction}

Oral squamous cell carcinoma (OSCC) is the most common head and neck cancer, and the overall prognosis of patients with OSCC remains poor. Because of extensive recent research on its pathogenesis and management, the 5-year survival rate for patients with OSCC has improved slightly within the last 15 years, but it still remains at $60 \%$ (1). A number of etiologic factors have been implicated in the development of OSCC, such as the use of tobacco and alcohol and the presence of incompatible prosthetic materials

Correspondence to: Dr Takao Setoguchi, Department of Orthopaedic Surgery, Graduate School of Medical and Dental Sciences, Kagoshima University, 8-35-1 Sakuragaoka, Kagoshima 890-8520, Japan

E-mail: setoro@m2.kufm.kagoshima-u.ac.jp

Key words: oral squamous cell carcinoma, Notch, $\gamma$-secretase inhibitor
(2). However, some patients develop OSCC without risk factors, which suggest that host susceptibility may also play a role in the development of OSCC. Molecular alterations in a number of oncogenes and tumor suppressor genes associated with the development of OSCC could be important for improving early detection and effective treatment of OSCC (3).

Members of the Notch family are highly conserved transmembrane receptors that influence the proliferation and apoptosis of diverse types of cells in a variety of organisms (4). Activation of Notch signaling requires binding of its ligands, Jagged and Delta-like (DLL) $(5,6)$, followed by proteolytic release of the Notch intracellular domain (NIC) and its translocation to the nucleus (7). NIC interacts with CSL, CBF1/RBP-Jк, Su (H), Lag-1, transcription factors and converts them from repressors to activators, thereby promoting transcription of downstream genes involved in various differentiation programs (8).

Recent studies have demonstrated constitutive activation of the Notch pathway in various types of malignancies. The oncogenic potential of Notch has been reported in human T-cell acute lymphoblastic leukemia (9), non-small cell lung cancer (10), ovarian carcinomas (11), colon cancer (12), pancreatic cancer (13) and osteosarcoma (14). On the other hand, Notch signaling promotes differentiation in skin cells (15), and loss of function of Notch is permissive for tumor formation in a mouse model (16). Although 3 expression array studies reported the upregulation of some members of the Notch signaling pathway in head and neck SCC including OSCC (17-19), the function of the Notch pathway in oral squamous cell tumorigenesis has not been previously characterized.

To explore the involvement of aberrant Notch signaling in the pathogenesis of OSCC, we investigated the expression of the Notch pathway molecules in both OSCC cell lines and biopsy specimens of OSCC patients, and we examined the effects of Notch pathway inhibition by a $\gamma$-secretase inhibitor (GSI), a pharmacological agent known to effectively block Notch activation by inhibiting the proteolysis and translocation of NIC to the nucleus. We found that Notchl, Notch2, Jagged1 and the Notch targets HES1 and HEY1 $(20,21)$ were upregulated in cells in both OSCC cell lines and biopsy specimens. In addition, $\gamma$-secretase inhibition prevented the growth of OSCC cells in vitro. 
Table I. PCR primer sequences.

\begin{tabular}{lll}
\hline Target molecules & & Primer sequence \\
\hline Notch1 & Forward & 5'-GTGACTGCTCCCTCAACTTCAAT-3' \\
& Reverse & 5'-CTGTCACAGTGGCCGTCACT-3' \\
Notch2 & Forward & 5'-AAAAATGGGGCCAACCGAGAC-3' \\
& Reverse & 5'-TTCATCCAGAAGGCGCACAA-3' \\
Jagged1 & Forward & 5'-CGGGATTTGGTTAATGGTTATC-3' \\
& Reverse & 5'-ATAGTCACTGGCACGGTTGTAGCAC-3' \\
HES1 & Forward & 5'-AGGCGGACATTCTGGAAATG-3' \\
& Reverse & 5'-CGGTACTTCCCCAGCACACTT-3' \\
HEY1 & Forward & 5'-CGAGGTGGAGAAGGAGAGTG-3' \\
& Reverse & 5'-CTGGGTACCAGCCTTCTCAG-3' \\
GAPDH & Forward & 5'-GAAGGTGAAGGTCGGAGTC-3' \\
& Reverse & 5'-GAAGATGGTGATGGGATTTC-3' \\
\hline
\end{tabular}

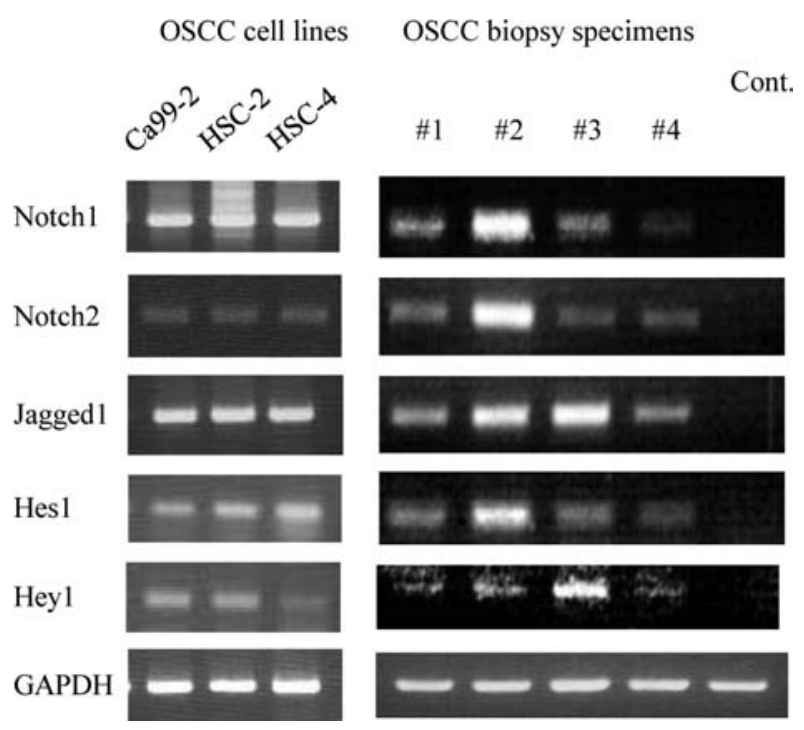

Figure 1. Expression of Notch signaling molecules in OSCC. RT-PCR revealed upregulation of Notch-related genes. Jagged1 is a Notch ligand; HES1 and HEY1 are Notch target genes.

\section{Materials and methods}

Cell culture. The human OSCC cell lines Ca99-2, HSC-2 and HSC-4 were purchased from Health Science Research Resources Bank (Osaka, Japan) and cultured in Dulbecco's modified Eagle's medium (Mediatech, Herndon, VA, USA) supplemented with $10 \%$ fetal bovine serum, penicillin $(100 \mathrm{U} / \mathrm{ml})$, and streptomycin $(100 \mu \mathrm{g} / \mathrm{ml})$. All cells were grown in a humidified atmosphere containing $5 \% \mathrm{CO}_{2}$ at $37^{\circ} \mathrm{C}$.

Biopsy specimens. All human OSCC biopsy specimens were obtained from primary lesions after informed consent according to a protocol that was approved by the institutional review board of Kagoshima University. Biopsies were performed before chemotherapy or radiotherapy to make the diagnosis. The respected tissues were divided into two parts: one was frozen immediately and stored at $-80^{\circ} \mathrm{C}$ until use, and the other was fixed in $10 \%$ buffered formaldehyde solution. Normal mucosa tissue used as a control was obtained from the tongue of a 76-year-old woman.

RT-PCR and real-time PCR. Total RNA was DNAse-treated and reverse-transcribed using oligo (dT) primers according to the manufacturer's protocol (Invitrogen, Carlsbad, CA, USA). cDNAs were synthesized with $5 \mu \mathrm{g}$ of total RNA as a template in a $20 \mu \mathrm{l}$ reaction mixture by using Superscript II reverse transcriptase (Gibco-BRL). After reverse transcription, each reaction mixture was diluted 5-fold with $\mathrm{H}_{2} \mathrm{O}$, and $1 \mu \mathrm{l}$ of each mixture was subjected to PCR using Taq polymerase (Takara, Tokyo, Japan). For real-time PCR assay, reactions were run using SYBR-Green (Bio-Rad, Hercules, CA, USA) on a MiniOpticon ${ }^{\mathrm{TM}}$ machine (Bio-Rad). The comparative $\mathrm{Ct}$ $(\Delta \Delta \mathrm{Ct})$ method was used to determine fold change in expression using BII-microglobulin. Each sample was run at three concentrations in triplicate. All primer sets amplified 100 to $200 \mathrm{bp}$ fragments. The primers sequences used are shown in Table I. The cycling conditions were as follows: initial denaturation at $98^{\circ} \mathrm{C}$ for $3 \mathrm{~min}$; 30 cycles at $98^{\circ} \mathrm{C}$ for $10 \mathrm{sec}, 60^{\circ} \mathrm{C}$ for $30 \mathrm{sec}$, and $72^{\circ} \mathrm{C}$ for $1 \mathrm{~min}$; and $72^{\circ} \mathrm{C}$ for $7 \mathrm{~min}$.

Immunohistochemical examination. HSC-4 cells were fixed with $4 \%$ paraformaldehyde in PBS and subjected to immunofluorescent staining. Cells were blocked with PBS containing $10 \%$ FCS and $0.1 \%$ Triton X-100. The following primary rabbit polyclonal antibodies were used: anti-NIC (diluted 1:200; Calbiochem, Basel, Switzerland) and antiJagged1 (diluted 1:200; R\&D Systems, Minneapolis, MN, USA). A fluorescent rhodamine-conjugated donkey antirabbit IgG antibody (diluted 1:200; Chemicon, Temecula, CA, USA) was used as the secondary antibody. The cells were counterstained with Hoechst 33258 (Molecular Probes, Carlsbad, CA, USA) to identify the nuclei. Immunohistochemistry with each secondary antibody alone without primary antibody was performed as a control. 
Notch-IC

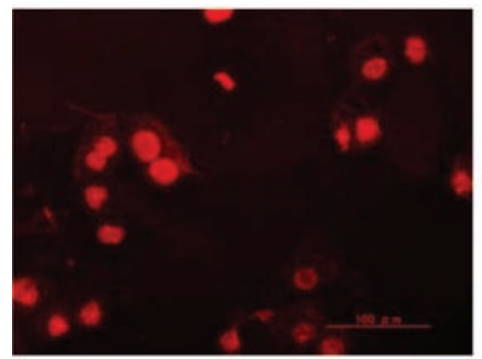

Jagged 1

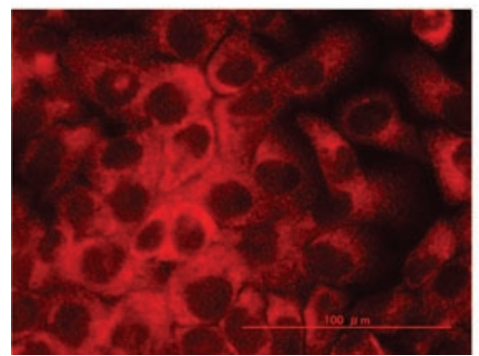

Hoechst

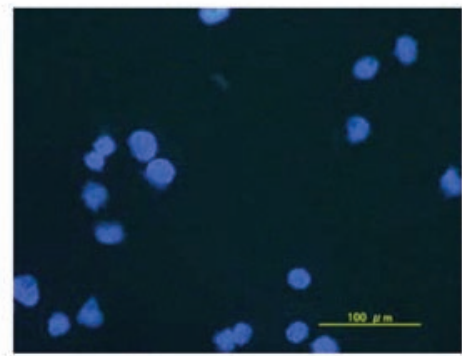

Hoechst

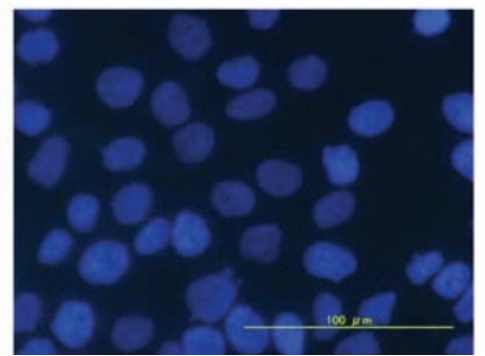

Merge

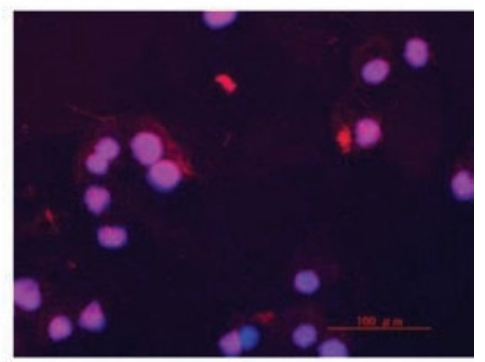

Merge

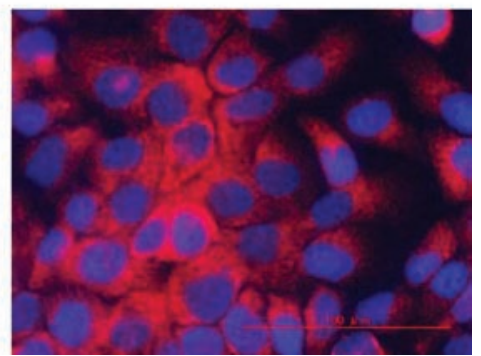

Figure 2. Expression of Notch intercellular domain (NIC) and Jagged1 in an OSCC cell line. Immunohistochemical examination revealed that NIC is accumulated in the nucleus of cells in OSCC cell lines, and Jagged1 is expressed in the cytoplasm of cells in OSCC cell lines.

Biopsy specimens were fixed routinely in $10 \%$ formalin, embedded in paraffin, and sectioned into $4-\mu \mathrm{m}$ thick slices. The immunostaining procedure was performed with the Evision kit/HRP (Dako, Glostrup, Denmark). In preparation for staining with anti-NIC and anti-Jagged1 antibodies, specimens were treated with $0.01 \mathrm{M}$ citrate buffer $(\mathrm{pH}$ 6.0) for $10 \mathrm{~min}$ at $121^{\circ} \mathrm{C}$ in an autoclave. The sections were then washed thrice with PBS and incubated with either rabbit polyclonal anti-NIC antibody (diluted 1:150; Cell Signaling Technology, Danvers, MA, USA) or rabbit polyclonal antiJagged1 antibody (diluted 1:50; Santa Cruz Biotechnology, Santa Cruz, CA, USA) diluted in PBS at $4^{\circ} \mathrm{C}$ overnight. After incubation with primary antibodies, the sections were incubated with the appropriate secondary antibodies conjugated with peroxidase-labeled dextran polymers for 40 min. After rinsing in PBS, the immune complex was visualized by incubating the sections with diaminobenzine tetrahydrochroride. The sections were rinsed briefly in water, counterstained with Mayer's hematoxylin, dehydrated, and mounted. Negative controls for the immunostaining procedure were prepared by omitting the primary antibody.

MTT assay. Cells were incubated with the substrate MTT [3(4,5-dimethylthiazol-2-yl)-2,5-diphenyltetrazolium bromide] for $4 \mathrm{~h}$, washed with PBS, and lysed to release formazan from cells. The cells were then analyzed in a Safire microplate reader (Bio-Rad) at $562 \mathrm{~nm}$. GSI X (Calbiochem, Basel, Switzerland) was used for the MTT assay.

\section{Results}

Upregulation of Notch pathway molecules in human OSCC. RT-PCR revealed that all OSCC cell lines and biopsy specimens expressed Notch1 and Notch2 mRNA (Fig. 1). In contrast, neither Notch1 nor Notch2 were expressed in the normal tongue tissue (Fig. 1). To further examine the expression of Notch pathway molecules, we performed RTPCR for Notch ligands and Notch target genes. Jaggedl was expressed in all OSCC cell lines and biopsy specimens, but not expressed in the normal tongue tissue (Fig. 1). Both HES1 and HEY1 mRNA were expressed in all cell lines and biopsy specimens, but neither of them was observed in the normal tongue tissue (Fig. 1). Immunocytochemical examination revealed that NIC was accumulated in the nuclei of HSC-4 cells, and Jagged1 was expressed in the cytoplasm of these cells (Fig. 2). We further investigated the expression of NIC and Jagged1 protein in biopsy specimens from OSCC patients. Immunohistochemical examination revealed that NIC was accumulated in the nuclei and Jagged1 was expressed in the cytoplasm of tumor cells from patients' biopsy specimens as well as in cell lines (Fig. 3). These findings suggest that the Notch signaling pathway is activated in human OSCC.

Inhibition of the Notch pathway prevents OSCC growth in vitro. To determine whether Notch pathway activation is required for OSCC cell growth, we used GSI X. We performed real-time PCR to determine the concentration of GSI $\mathrm{X}$ that effectively inhibited Notch activity in OSCC cells, and then measured the expression of the Notch pathway target gene HES1. In HSC-2 and HSC-4 cells, GSI X at a concentration of $5 \mu \mathrm{M}$ was found to reduce mRNA levels of HES1, particularly in HSC-4 cells in which HES1 decreased by more than $90 \%$ (Fig. 4A). Because GSI X prevented osteosarcoma cell growth at $5 \mu \mathrm{M}$ concentration (14), we decided that $5 \mu \mathrm{M}$ was an appropriate concentration for 


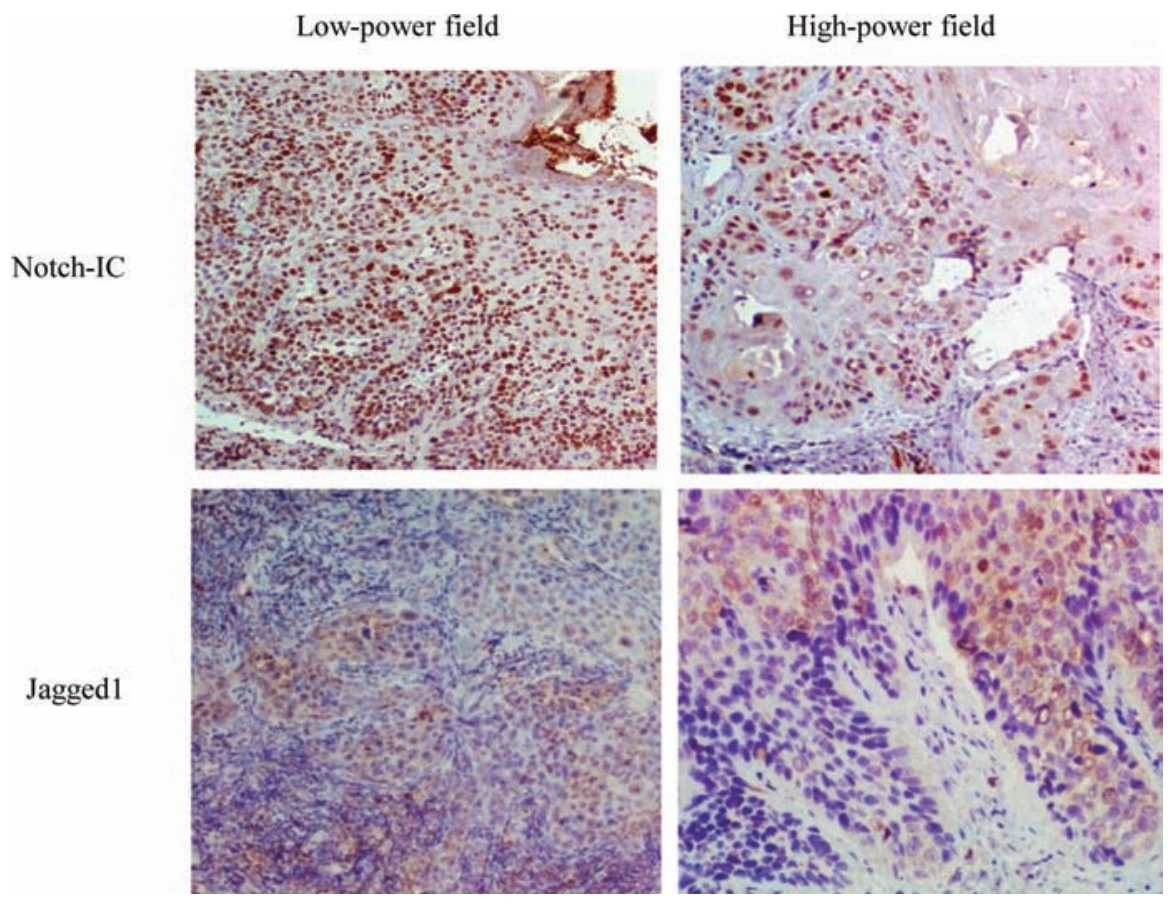

Figure 3. Expression of Notch intercellular domain (NIC) and Jagged1 in biopsy specimens from OSCC patients. Immunohistochemical examination revealed that NIC is accumulated in the nucleus of tumor cells in OSCC biopsy specimens, and Jagged1 is expressed in the cytoplasm of tumor cells in OSCC biopsy specimens. These results suggest that Notch signaling is activated in tumor cells in biopsy specimens from OSCC patients.

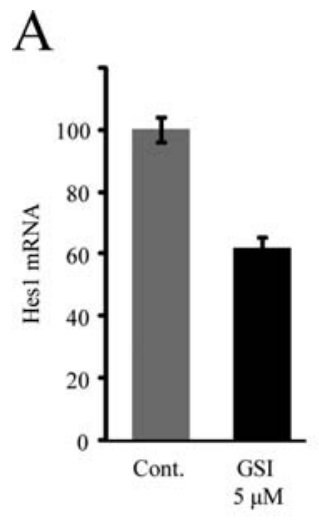

HSC-2

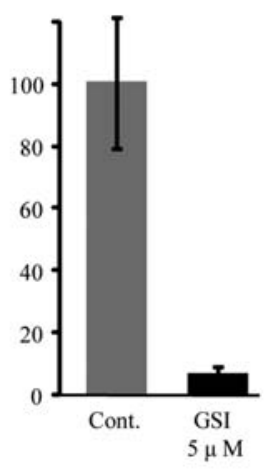

HSC-4

B
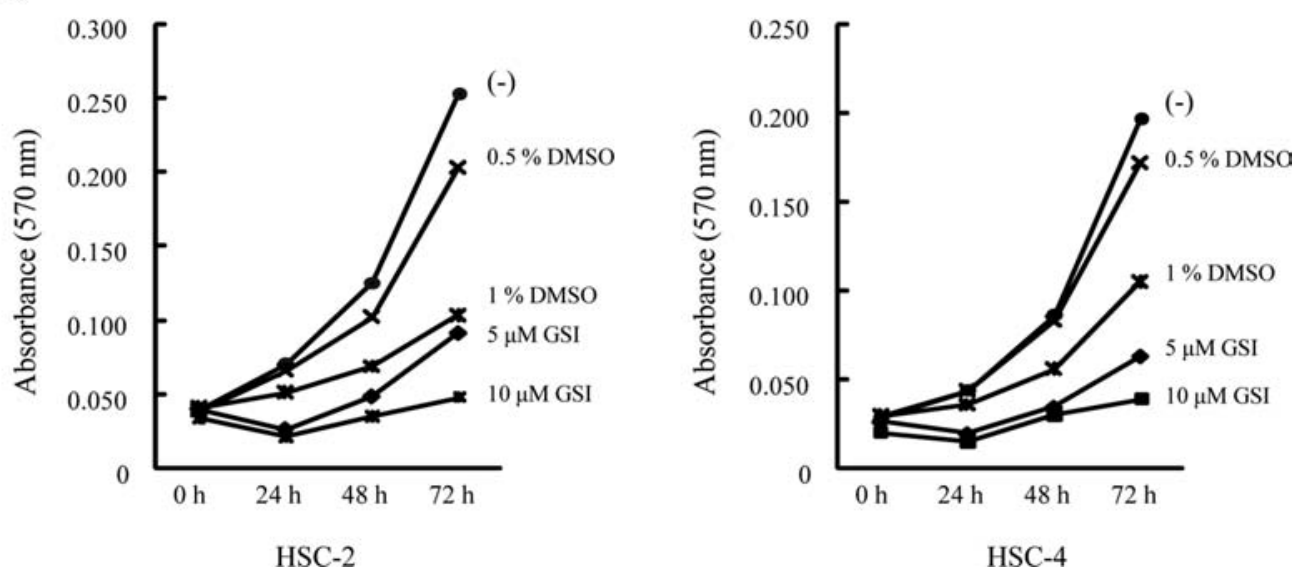

Figure 4. Inhibition of the Notch pathway prevents growth in OSCC cell lines. We performed real-time PCR to determine the concentration of GSI X that effectively inhibited Notch activity in OSCC cells, and then measured the expression of the Notch pathway target gene HES1. (A) GSI X at $5 \mu$ M reduced mRNA levels of HES1 in HSC-2 and HSC-4 cells (error bar indicates standard deviation). (B) Growth of viable HSC-2 and HSC-4 cells over 3 days was slowed by GSI $\mathrm{X}$ in a dose-dependent fashion. 
treating OSCC. We then assessed tumor growth in vitro under GSI treatment. The MTT assay revealed that GSI treatment slowed the growth of HSC-2 and HSC-4 cells in a dose-dependent fashion (Fig. 4B).

\section{Discussion}

Deregulation of Notch signaling is implicated in the development of various cancers, and Notch blockade appears to affect the survival and proliferation of multiple types of cancers (22-24). For example, Notch is activated by translocation or mutation in more than half of T-cell acute lymphoblastic leukemias, and anti-Notch treatments have been shown to slow the growth of acute lymphoblastic leukemia in vitro (25). In addition, combining GSIs, which inhibit Notch signaling, with anticancer drugs improves their anticancer effects $(26,27)$.

We examined the expression of the Notch pathway genes in cell lines and tumors, and found that the expression levels of Notch1, Notch2, Jagged1, HES1 and HEY1 were upregulated compared to those of these genes in the normal oral tissue, suggesting that Notch signaling is active in OSCC. A number of previous studies support these findings and indicate a role for deregulation of Notch signaling in OSCC. Köse et al reported that Notch1 was absent in normal oral mucosa and even in oral pre-cancer lesions (28). Zeng et al revealed that Jagged1 was highly expressed in OSCC compared to that in the normal oral tissue, and Jagged1-expressing SCC cells were surrounded by microvessels (29). In recent expression array studies, two groups have independently reported significant upregulation of Jaggedl and Notch4 in OSCC compared to that in the normal oral tissue $(17,18)$. In addition, Snijders et al have reported that DLL1 and RBPSUH are upregulated in OSCC (19).

GSIs prevent Notch receptor activation, and several forms of GSI have been tested for their antitumor effects (30). GSIs induce cell-cycle arrest and apoptosis in multiple cancer cell lines (26,31-33). We examined whether inhibition of the Notch pathway using GSI can block Notch signaling in OSCC and reduce tumor growth in vitro. Treatment of OSCC cells with GSI effectively reduced HESI mRNA and prevented growth in OSCC cell lines. These findings suggest that GSIs effectively inhibit the Notch pathway in OSCC cell lines and indicate an association between the activation of Notch signaling and pro-oncogenic effects in human OSCC. The role of GSI in inhibiting the growth of OSCC demonstrated in the present study is consistent with the previous findings in other malignancies (14,26,27,31,34-36).

In summary, our findings demonstrate that the Notch signaling pathway is functionally activated in human OSCC. This novel finding adds to the understanding of OSCC and may be important in understanding the proliferation of OSCC cells. Furthermore, the finding of growth inhibition by GSI, a Notch inhibitor, suggests that inactivation of Notch may be a useful approach for the treatment of patients with OSCC.

\section{Acknowledgements}

This work was supported by Grants-in-Aid for Scientific Research (B) 18390419 and (C) 19591725 (KAKENHI).

\section{References}

1. Jemal A, Siegel R, Ward E, et al: Cancer statistics, 2008. CA Cancer J Clin 58: 71-96, 2008.

2. Scully C and Bagan JV: Recent advances in Oral Oncology. Oral Oncol 43: 107-115, 2007.

3. Nagpal JK and Das BR: Oral cancer: reviewing the present understanding of its molecular mechanism and exploring the future directions for its effective management. Oral Oncol 39: 213-221, 2003.

4. Artavanis-Tsakonas S, Rand MD and Lake RJ: Notch signaling: cell fate control and signal integration in development. Science 284: 770-776, 1999.

5. Bettenhausen B, Hrabe de Angelis M, Simon D, Guenet JL and Gossler A: Transient and restricted expression during mouse embryogenesis of Dll1, a murine gene closely related to Drosophila Delta. Development 121: 2407-2418, 1995.

6. Oda T, Elkahloun AG, Pike BL, et al: Mutations in the human Jagged1 gene are responsible for Alagille syndrome. Nat Genet 16: 235-242, 1997.

7. De Strooper B, Annaert W, Cupers $\mathrm{P}$, et al: A presenilin-1dependent gamma-secretase-like protease mediates release of Notch intracellular domain. Nature 398: 518-522, 1999.

8. Davis RL and Turner DL: Vertebrate hairy and Enhancer of split related proteins: transcriptional repressors regulating cellular differentiation and embryonic pat-terning. Oncogene 20: 8342-8357, 2001.

9. Grabher C, von Boehmer H and Look AT: Notch 1 activation in the molecular pathogenesis of T-cell acute lymphoblastic leukaemia. Nat Rev Cancer 6: 347-359, 2006.

10. Dang TP, Gazdar AF, Virmani AK, et al: Chromosome 19 translocation, overexpression of Notch3, and human lung cancer. J Natl Cancer Inst 92: 1355-1357, 2000.

11. Park JT, Li M, Nakayama K, et al: Notch3 gene amplification in ovarian cancer. Cancer Res 66: 6312-6318, 2006.

12. Reedijk M, Odorcic S, Zhang H, et al: Activation of Notch signaling in human colon adenocarcinoma. Int J Oncol 33: 1223-1229, 2008.

13. Miyamoto Y, Maitra A, Ghosh B, et al: Notch mediates TGF alpha-induced changes in epithelial differentiation during pancreatic tumorigenesis. Cancer Cell 3: 565-576, 2003.

14. Tanaka M, Setoguchi T, Hirotsu M, et al: Inhibition of Notch pathway prevents osteosarcoma growth by cell cycle regulation. Br J Cancer 100: 1957-1965, 2009.

15. Rangarajan A, Talora C, Okuyama R, et al: Notch signaling is a direct determinant of keratinocyte growth arrest and entry into differentiation. EMBO J 20: 3427-3436, 2001.

16. Nicolas M, Wolfer A, Raj K, et al: Notch1 functions as a tumor suppressor in mouse skin. Nat Genet 33: 416-421, 2003.

17. Ha PK, Benoit NE, Yochem R, et al: A transcriptional progression model for head and neck cancer. Clin Cancer Res 9: 3058-3064, 2003.

18. Leethanakul C, Patel V, Gillespie J, et al: Distinct pattern of expression of differentiation and growth-related genes in squamous cell carcinomas of the head and neck revealed by the use of laser capture microdissection and cDNA arrays. Oncogene 19: 3220-3224, 2000.

19. Snijders AM, Schmidt BL, Fridlyand J, et al: Rare amplicons implicate frequent deregulation of cell fate specification pathways in oral squamous cell carcinoma. Oncogene 24: 4232-4242, 2005.

20. Jarriault S, Brou C, Logeat F, Schroeter EH, Kopan R and Israel A: Signalling downstream of activated mammalian Notch. Nature 377: 355-358, 1995.

21. Maier MM and Gessler M: Comparative analysis of the human and mouse Hey 1 promoter: Hey genes are new Notch target genes. Biochem Biophys Res Commun 275: 652-660, 2000.

22. Radtke F and Raj K: The role of Notch in tumorigenesis: oncogene or tumour suppressor? Nat Rev Cancer 3: 756-767, 2003.

23. Politi K, Feirt N and Kitajewski J: Notch in mammary gland development and breast cancer. Semin Cancer Biol 14: 341-347, 2004.

24. Weng AP and Aster JC: Multiple niches for Notch in cancer: context is everything. Curr Opin Genet Dev 14: 48-54, 2004.

25. Weng AP, Ferrando AA, Lee W, et al: Activating mutations of NOTCH1 in human T cell acute lymphoblastic leukemia. Science 306: 269-271, 2004.

26. Meng RD, Shelton CC, Li YM, et al: gamma-Secretase inhibitors abrogate ox-aliplatin-induced activation of the Notch-1 signaling pathway in colon cancer cells resulting in enhanced chemosensitivity. Cancer Res 69: 573-582, 2009. 
27. Real PJ, Tosello V, Palomero T, et al: Gamma-secretase inhibitors reverse glucocorticoid resistance in $\mathrm{T}$ cell acute lymphoblastic leukemia. Nat Med 15: 50-58, 2009.

28. Kose O, Lalli A, Kutulola AO, Odell EW and Waseem A: Changes in the expression of stem cell markers in oral lichen planus and hyperkeratotic lesions. J Oral Sci 49: 133-139, 2007.

29. Zeng Q, Li S, Chepeha DB, et al: Crosstalk between tumor and endothelial cells promotes tumor angiogenesis by MAPK activation of Notch signaling. Cancer Cell 8: 13-23, 2005.

30. Shih Ie $\mathrm{M}$ and Wang TL: Notch signaling, gamma-secretase inhibitors, and cancer therapy. Cancer Res 67: 1879-1882, 2007.

31. Curry CL, Reed LL, Golde TE, Miele L, Nickoloff BJ and Foreman KE: Gamma secretase inhibitor blocks Notch activation and induces apoptosis in Kaposi's sarcoma tumor cells. Oncogene 24: 6333-6344, 2005.

32. Kogoshi H, Sato T, Koyama T, Nara N and Tohda S: $\gamma$-secretase inhibitors suppress the growth of leukemia and lymphoma cells. Oncol Rep 18: 77-80, 2007.
33. Konishi J, Kawaguchi KS, Vo H, et al: Gamma-secretase inhibitor prevents Notch3 activation and reduces proliferation in human lung cancers. Cancer Res 67: 8051-8057, 2007.

34. Pece S, Serresi M, Santolini E, et al: Loss of negative regulation by Numb over Notch is relevant to human breast carcinogenesis. J Cell Biol 167: 215-221, 2004.

35. Park JT, Shih Ie M and Wang TL: Identification of Pbx1, a potential oncogene, as a Notch3 target gene in ovarian cancer. Cancer Res 68: 8852-8860, 2008.

36. Bin Hafeez B, Adhami VM, Asim M, et al: Targeted knockdown of Notch1 inhibits invasion of human prostate cancer cells concomitant with inhibition of matrix metalloproteinase- 9 and urokinase plasminogen activator. Clin Cancer Res 15: 452-459, 2009. 